\title{
Bilayer Properties of 1,3-Diamidophospholipids
}

\author{
Andreas Weinberger, ${ }^{\dagger}$ Radu Tanasescu, ${ }^{\dagger}$ Cristina Stefaniu, ${ }^{\S}$ lllya A. Fedotenko," France Favarger," \\ Takashi Ishikawa, ${ }^{\mathcal{1}}$ Gerald Brezesinski, ${ }^{*, \$}$ Carlos M. Marques, ${ }^{*, \#}$ and Andreas Zumbuehl ${ }^{*,+, *}$ \\ $\dagger$ Department of Chemistry, University of Fribourg, Chemin du Musée 9, 1700 Fribourg, Switzerland \\ ${ }^{+}$National Center of Competence in Research in Chemical Biology, 1211 Geneva, Switzerland \\ ${ }^{\S}$ Max Planck Institute of Colloids and Interfaces, Research Campus Potsdam-Golm, 14476 Potsdam, Germany \\ "Department of Organic Chemistry, University of Geneva, Quai Ernest-Ansermet 30, 1211 Geneva, Switzerland \\ ${ }^{\perp}$ Paul Scherrer Institute (PSI), OFLB/010, 5232 Villigen PSI, Switzerland \\ \#Institut Charles Sadron (CNRS-UPR 22), Université de Strasbourg, Rue du Loess 23, 67034 Strasbourg, France
}

\begin{abstract}
A series of 1,3-diamido phosphocholines was synthesized, and their potential to form stable bilayers was investigated. Large and giant unilamellar vesicles produced from these new lipids form a wide variety of faceted liposomes. Factors such as cooling rates and the careful choice of the liposome preparation method influence the formation of facets. Interdigitation was hypothesized as a main factor for the stabilization of facets and effectively monitored by small-angle $\mathrm{X}$-ray scattering measurements.
\end{abstract}

\section{INTRODUCTION}

The unique potential of liposomes for drug delivery ${ }^{1}$ was recognized very shortly after their discovery almost 50 years ago. ${ }^{2}$ Nowadays, liposomal drugs are a standard player in pharmaceutical research. One of the primary goals of drug delivery is to maximize the therapeutic effect of the drug together with the minimization of its side-effects. ${ }^{3}$ The ability to entrap a cargo in the interior of liposomes or the bilayer, combined with the ability for a triggered release by variation of parameters such as $\mathrm{pH}$, temperature, and shear stress, ${ }^{4}$ allows researchers to achieve this goal. Originally, liposomes were mainly formed spontaneously from natural occurring lipids, but in the past years artificial lipids were also investigated as potential candidates for smart drug delivery systems.

Recently, liposomes prepared from an artificial $\mathrm{C}_{16^{-1,3}}$ diamidophospholipid have been shown to display mechanosensitive properties. ${ }^{5}$ The origin of such shear-sensitivity has however not been yet identified. This artificial 1,3-diaminophospholipid, named Pad-PC-Pad (nomenclature in Fedotenko et al. ${ }^{6}$ ), forms faceted vesicles, which leads to discontinuous membranes with defects. ${ }^{7}$ The attenuation of these defects during mechanical stimulation of the vesicles might be a possible reason for the mechanosensitive properties.

When liposomes of natural phospholipids are formed, they typically possess spherical shapes. However, already 30 years ago it was noted that small unilamellar vesicles (SUV) of $40 \mathrm{~nm}$ diameter formulated from 1,2-dipalmitoyl-phosphatidylcholine (DPPC) are most probably faceted below the gel-to-liquidcrystalline phase transition temperature $T_{\mathrm{m}}{ }^{8}$ but the high curvature found in SUVs leads to packing disorders that

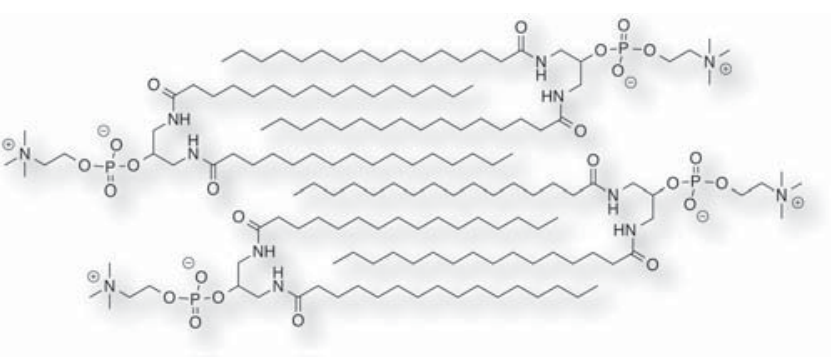

resulted in spontaneous vesicle fusion into large unilamellar spherical vesicles., ${ }^{9,10}$ Furthermore, even giant unilamellar vesicles (GUVs) can be faceted either spontaneously, ${ }^{11}$ or when undergoing a phase transition. ${ }^{12}$ At the beginning of this century, the discussion on faceted soft matter constructs was revived by several reports on nonspherical vesicles spontaneously formed from catanionic surfactants ${ }^{13}$ or nonionic surfactants ${ }^{14}$ or triggered by the addition of polyelectrolytes. ${ }^{15}$ Other theoretical and experimental papers followed in recent years, ${ }^{16-21}$ reporting polyhedral shapes even for vesicles with fluid membranes. $^{22}$

Recently, we have noted that liposomes formulated from the symmetric, artificial 1,3-diamidophospholipid Pad-PC-Pad, even though significantly larger than $40 \mathrm{~nm}$, form faceted large unilamellar vesicles (LUVs) and GUVs below the main phase transition. ${ }^{5}$ Facets have not been reported for spontaneously formed LUVs (larger than $100 \mathrm{~nm}$ ) or GUVs of single component phospholipids. Monolayer studies performed by Fedotenko et al. ${ }^{23}$ showed that 1,3-diamidophospolipids possess strong hydrogen-bonding headgroup interactions, which could be responsible for the observed faceted shapes. In this study a much lower critical temperature of the monolayers, as compared to the main phase transition temperature of the bilayers, was observed. The authors suggested that this could be explained by membrane interdigitation. 
Membrane interdigitation was previously observed in phospholipid bilayers. For most of the systems of equal chain-length phospholipids it was caused by addition of active agents, such as alcohols, ${ }^{24-27}$ surface active molecules, ${ }^{28}$ anesthetics, ${ }^{29}$ or semifluorinated alkanes. ${ }^{30}$ Interdigitation was also observed in bilayers from mixed chain-length phospholipids. $^{31,32}$ 1,2-Di-O-hexadecyl-sn-glycero-3-phosphocholine (DHPC) for instance is one of the few phospholipids which has equal tail-length and spontaneously interdigitates. ${ }^{33}$ Serrallach et al. $^{34}$ showed that interdigitation occurs, when the 1,2 linkage to the glycerol backbone in $\alpha$-DPPC is switched to a 1,3 linkage as in $\beta$-DPPC. 1,3 -DPPC can exist in a fully interdigitated gel structure at temperatures below $T_{\mathrm{m}}$ and can form a noninterdigitated phase for even lower temperatures.

Recently, we have reported on the monolayer properties of 1,3-diamidophospholipids. ${ }^{23}$ Here we expanded our studies to the bilayer properties of a small library of the Xad-PC-Xad genus of phospholipids with tails ranging from $C_{12}$ to $C_{18}$ (see Figure 1). The goal was to investigate if other types of 1,3-

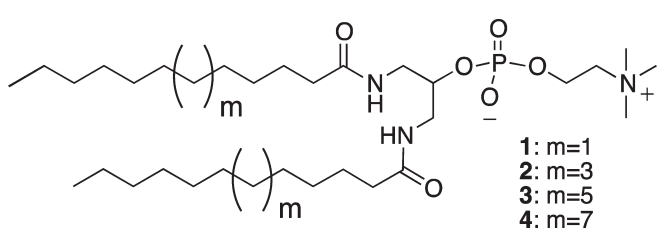

Figure 1. Xad-PC-Xad genus of 1,3-diamido phospholipids: Lad-PCLad (1), Mad-PC-Mad (2), Pad-PC-Pad (3), and Sad-PC-Sad (4).

diamidophospholids behave similar to Pad-PC-Pad and to elaborate on the question of why stable faceted vesicles are formed and if interdigitation might play a role in the formation of facets.

\section{RESULTS AND DISCUSSION}

Large unilamellar vesicles formed by extrusion through $100 \mathrm{~nm}$ diameter pores $\left(\right.$ LUVET $\left._{100}\right)$ from Pad-PC-Pad (3) showed a distinctive lenticular shape in the cryo-TEM. ${ }^{5}$ We hypothesized that this is due to an intermolecular hydrogen-bonding network at the hydrophobic-hydrophilic interface of the lipids and due to possible membrane interdigitation. Pad-PC-Pad (3) is in the gel state $\left(T_{\mathrm{m}} \approx 35{ }^{\circ} \mathrm{C}\right)$ at room temperature. ${ }^{5}$ The main transition temperature of our small 1,3-diamidophospholipid library is increasing linearly with the length of the hydrocarbon chain: $\mathrm{C}_{12}<0{ }^{\circ} \mathrm{C} ; \mathrm{C}_{14} \approx 21{ }^{\circ} \mathrm{C} ; \mathrm{C}_{16} \approx 37{ }^{\circ} \mathrm{C} ; \mathrm{C}_{18} \approx 52{ }^{\circ} \mathrm{C}$.

Therefore, Sad-PC-Sad (4) should also show a faceted morphology at room temperature, whereas Lad-PC-Lad (1) due to its much lower $T_{\mathrm{m}}$ should show no facets at all and MadPC-Mad (2) only at lower temperatures. Cryo-TEM micrographs were recorded according to an established protocol. ${ }^{35}$
Unilamellar and multilamellar vesicles with nonspherical morphology were indeed found (see Figure 2). Unfortunately, neither Lad-PC-Lad (1) nor Mad-PC-Mad (2) could be easily formulated into liposomes, as the suspensions were not stable, and a precipitate formed, a tendency also observed by DLS measurements in a smaller scale for Pad-PC-Pad vesicles during the course of several days. However, contrary to the Pad-PCPad liposomes, the precipitates of Lad-PC-Lad and Mad-PCMad cannot be resuspended to obtain nonaggregated liposomes.

Recently, Rothemund introduced the concept of DNA origami where DNA could be folded into any imaginable 3D structure. ${ }^{36}$ In the field of lipids, catanionic vesicles were found to organize into icosahedral structures in salt-free media, ${ }^{13}$ and sulfamide lipids formed GUVs from rigid $2 \mathrm{D}$ sheets. ${ }^{37}$ Figure 3
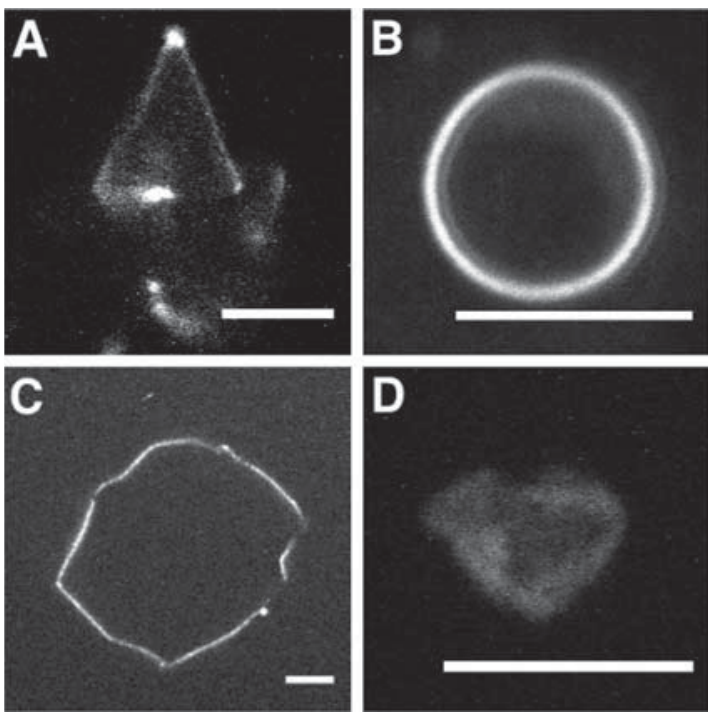

Figure 3. Giant unilamellar vesicles at $20{ }^{\circ} \mathrm{C}$ were prepared from 50 mol \% Sad-PC-Sad (4) and $50 \mathrm{~mol} \%$ Pad-PC-Pad (3, A and B), or 50 $\mathrm{mol} \%$ Sad-PC-Sad (4) and $50 \mathrm{~mol} \%$ Mad-PC-Mad (2, C), or $50 \mathrm{~mol}$ $\%$ Sad-PC-Sad (4) and $50 \mathrm{~mol} \%$ Lad-PC-Lad $(1, \mathrm{D})$. All preparations contain $1 \mathrm{~mol} \%$ of Rhodamine labeled DOPE. Scale bars $=5 \mu \mathrm{m}$.

shows that indeed our artificial phospholipids can be formulated into giant unilamellar vesicles of various morphologies by preparing mixtures thereof and thus controlling their morphologies. As described above, Lad-PC-Lad and Mad-PCMad could not be easily formulated into GUVs. In the case of lipid mixtures, GUVs of these lipids could be prepared with molar ratios of up to $50 \%$ of Lad-PC-Lad or Mad-PC-Mad.

Facet Formation: Kinetically Controlled? Artificial 1,3diamidophospholipids show faceted shapes below their main
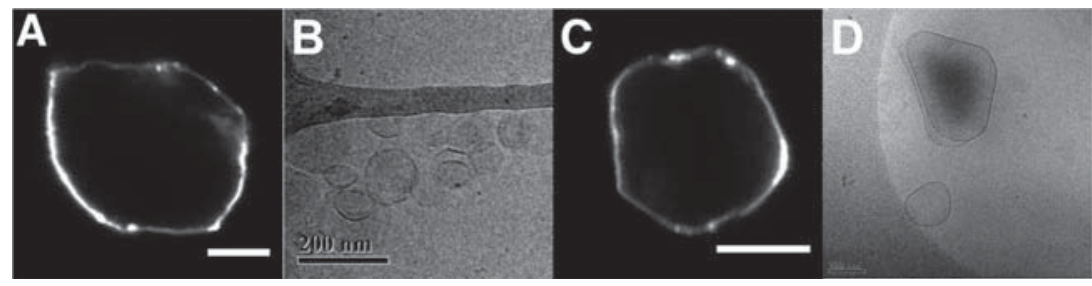

Figure 2. Pad-PC-Pad (3) micrographs of a GUV (A, fluorescent micrograph) and LUVs (B, cryo TEM). Sad-PC-Sad (4) micrographs of a GUV (C, fluorescent micrograph) and LUVs (D, cryo TEM). All GUV preparations contain $1 \mathrm{~mol} \%$ of Rhodamine labeled DOPE. Scale bars $=5 \mu \mathrm{m}$ for GUVs and $200 \mathrm{~nm}$ for LUVs. 
transition temperature. In order to investigate factors influencing the formation of facets, giant unilamellar vesicles (GUV) were prepared with the standard electroformation method using ITO slides. ${ }^{38}$ All preparations were done at 55 ${ }^{\circ} \mathrm{C}$, above the $T_{\mathrm{m}}$ of the lipids, and then cooled to $25{ }^{\circ} \mathrm{C}$ prior to confocal microscopy analysis. The pure lipid formulations of Pad-PC-Pad (3) and Sad-PC-Sad (4) showed faceted GUVs expected from lipids in the gel phase (see Figure 2).

However, judging from TLC and mass spectroscopy, the electroformation of Pad-PC-Pad led to a hydrolysis of the phosphate diester, and after $1 \mathrm{~h}$ of electroformation, only the free phosphate monoester Pad-POH-Pad was found. Therefore, some of the GUV morphologies found are probably induced by degraded phospholipids. Feigenson indeed noted that lipids can be hydrolyzed during electroformation on ITO glass slides. ${ }^{39}$

The serious problem encountered with the electroformation protocol led us to have a closer look at the phenomenon. Indeed, a GUV formulated from Pad-PC-Pad (3) via electroformation showed the typical domain structure often encountered in recent literature (see Figure 4A). However,

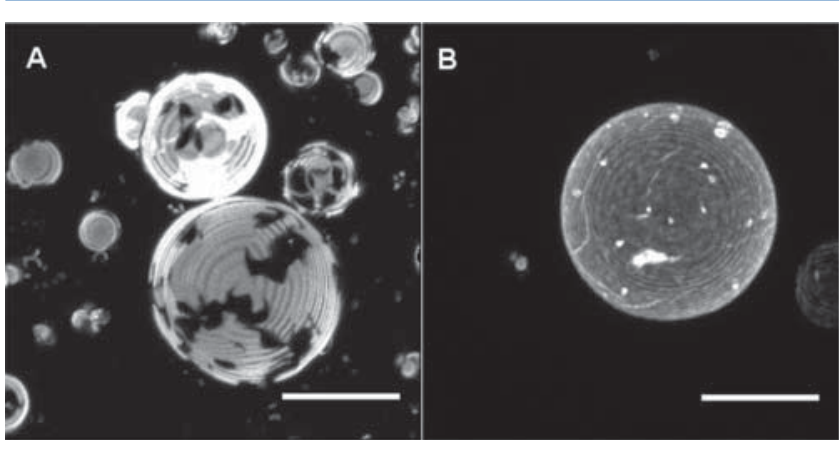

Figure 4. GUVs formed by the electroformation protocol (A) show typical domains. No such domains are found when a more gentle preparation protocol (rehydration of a multilayer of lipids on a hydrogel) was applied (B). This is corroborating the fact that some phospholipids undergo chemical reactions during electroformation. Scale bars $=20 \mu \mathrm{m}$.

such a biphasic system of one lipid at one temperature should not be possible. ${ }^{40}$ Indeed, no domains were found when a more gentle GUV preparation protocol was applied: the hydration of lipid multilayers on an agarose ${ }^{41}$ or PVA gel $^{42}$ (see Figure 4B). As a reference, no such degradation was found for the natural phospholipid DPPC in either case. It should be noted that the gentle hydration method for the formation of GUVs of Sad-PCSad is not optimal: Due to the high transition temperature of Sad-PC-Sad and the dissolution of the gel at temperatures above $50{ }^{\circ} \mathrm{C}$, only a few GUVs could be obtained, and they were contaminated by agarose or PVA gel fragments.

To ensure that facet formation is not induced by the presence of degraded lipids, the gentle hydration method was used for the further preparation of GUVs. ${ }^{42}$ If the GUVs of Xad-PC-Xad are prepared at temperatures above the corresponding $T_{\mathrm{m}}$ of the lipids, only rapid cooling $\left(\geq 4{ }^{\circ} \mathrm{C} /\right.$ $\min$ ) leads to faceted vesicles (see Figure $5 \mathrm{~A}$ ). When the GUVs have been cooled down more slowly $\left(\leq 0.5-1{ }^{\circ} \mathrm{C} / \mathrm{min}\right)$, they were spherical with no apparent facets (see Figure $5 \mathrm{~B}$ ). Moreover, in some cases it was found that rapid cooling of DPPC GUVs also yields faceted vesicles (see Figure 5C), and slow cooling leads to spherical vesicles (see Figure 5D). In any case, faceted vesicles of DPPC obtained by rapid cooling are
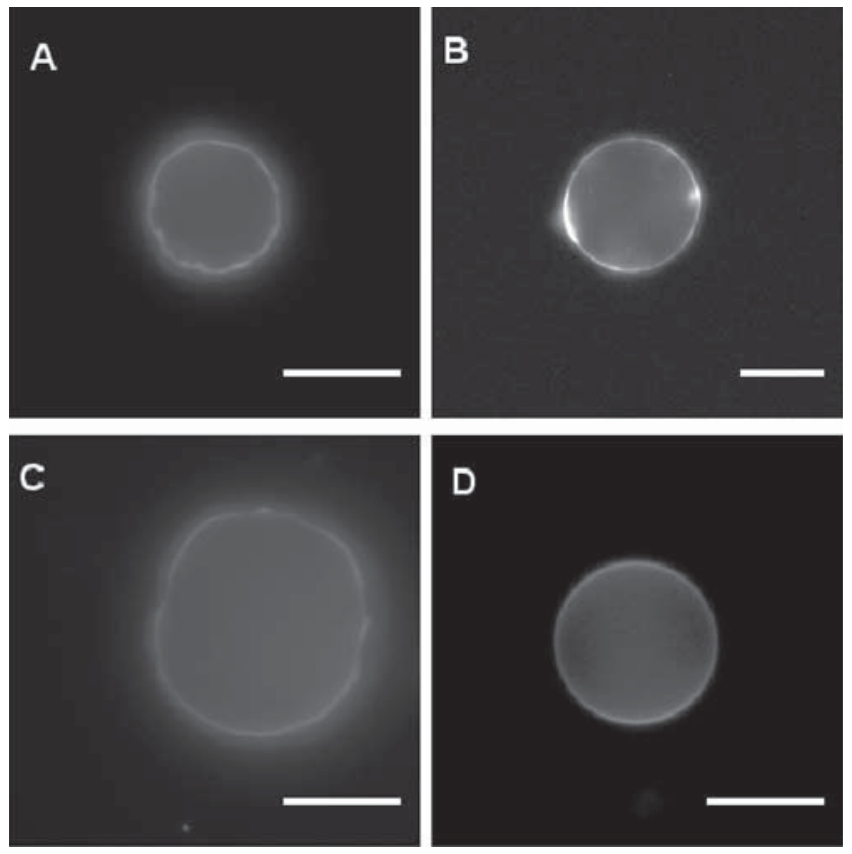

Figure 5. Formation of faceted GUVs depends on the rate of cooling after electroformation: rapid cooling $\left(\mathrm{A}, \geq 4{ }^{\circ} \mathrm{C} / \mathrm{min}\right)$ and slow cooling $\left(\mathrm{B}, \leq 0.5-1{ }^{\circ} \mathrm{C} / \mathrm{min}\right)$ of Pad-PC-Pad (3) and rapid cooling (C) and slow cooling (D) of DPPC. Scale bars $=20 \mu \mathrm{m}$. Only rapid cooling leads to faceted vesicles.

not stable and disappear within 15 min, whereas facets in PadPC-Pad vesicles are stable for more than $1 \mathrm{~h}$.

This behavior can be easily explained by the kinetic dependence of the conversion of a noninterdigitated $\mathrm{L}_{\alpha}$ phase to a (possible) interdigitated $\mathrm{L}_{\beta}{ }^{\prime}$ phase. ${ }^{34}$ In the literature several reports can be found that interdigitated bilayers cause a shift to higher main transition temperatures. ${ }^{33}$ This effect should be reproducible with DSC measurements of MLV suspensions with cooling rates in the range $0.5-2.0^{\circ} \mathrm{C} / \mathrm{min}$. A higher cooling rate was not possible due to the maximum possible cooling rate of the used DSC apparatus. Contrary to DPPC, Pad-PC-Pad forms faceted vesicles independently of its size and in all observed cases. Therefore, Pad-PC-Pad should show a shift in the main transition temperatures depending on the cooling rate.

The DSC curves showed a main transition at $41.8{ }^{\circ} \mathrm{C}$ for DPPC with a $\Delta H$ of $30.5 \mathrm{~kJ} / \mathrm{mol}$ and at $33.7^{\circ} \mathrm{C}$ for Pad-PCPad with a corresponding $\Delta H$ of $33.2 \mathrm{~kJ} / \mathrm{mol}$ (obtained from the cooling curves). The differences for the values obtained for $T_{\mathrm{m}}$ and $\Delta H$ for the different cooling rates were negligible. However, a difference in peak shape was found. A lower cooling rate leads to sharp peaks because the system has time to adopt to the phase change. For DPPC a pretransition peak at $30.2{ }^{\circ} \mathrm{C}$ was observed, whereas Pad-PC-Pad has no pretransition peak. This result might already indicate a possible interdigitation for Pad-PC-Pad, as literature results show that chemically induced interdigitation causes a disappearance of the pretransition peak and an increase in enthalpy. ${ }^{33}$ Figure 6 shows the recorded DSC curves of MLV solutions of DPPC and Pad-PC-Pad.

The measurement of proton permeability by fluorescence quenching is another method, which can be applied to characterize interdigitated bilayers. ${ }^{43}$ With this method a drastic increase of proton permeability was observed caused by interdigitation. In literature, an increase of proton permeability 


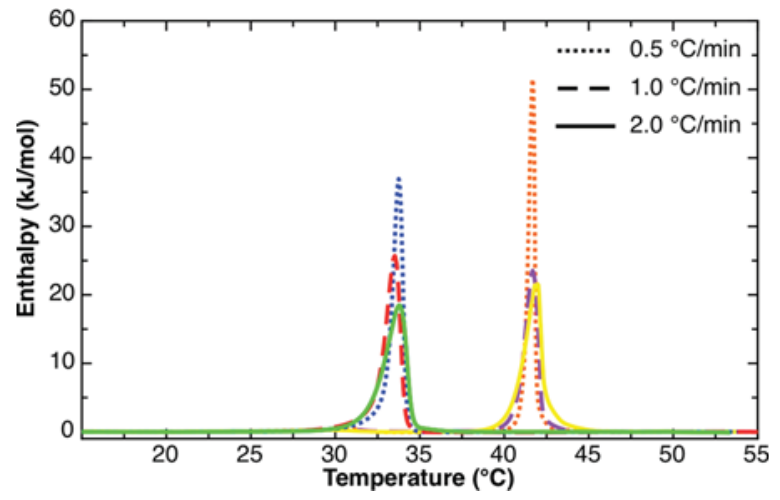

Figure 6. DSC cooling curves of MLV suspensions of Pad-PC-Pad at $33.7^{\circ} \mathrm{C}$ (left) and DPPC at $41.8^{\circ} \mathrm{C}$ (right) in water.

was also attributed to a coexistence of interdigitated and noninterdigitated phases. ${ }^{44}$

The aforementioned method was used to determine the proton permeability of LUVs from DPPC and Pad-PC-Pad by monitoring the fluorescence decrease of 5(6)-carboxyfluorescein over time after application of a $\mathrm{pH}$ shock from 7.4 to 3 . As reported previously, ${ }^{43}$ the curve follows an exponential decay and can be fitted to determine the rate constant by eq 1 :

$$
y=A \exp (-k t)+C
$$

The proton permeability constant can be calculated from the fitted rate constants and the vesicle radius of $100 \mathrm{~nm}$ by eq 2 .

$$
P=\frac{k R}{3}
$$

We obtained permeability coefficients for Pad-PC-Pad and DPPC in the range $10^{-4}$ to $10^{-5} \mu \mathrm{m} / \mathrm{s}$, comparable to previously reported permeability coefficients measured with this method. ${ }^{43,45}$ Typical quenching curves are shown in Figure 7.

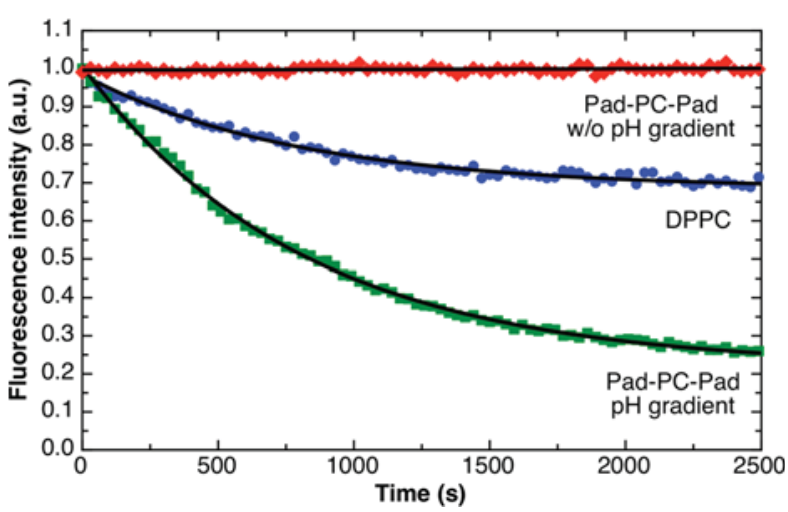

Figure 7. Evolution of the fluorescence intensity of carboxyfluorescein loaded LUVs of DPPC (blue circles) and Pad-PC-Pad (green squares) after application of a $\mathrm{pH}$ shock. Red diamonds show that entrapped 5(6)-carboxyfluorescein does not leak from LUVs and does not bleach during measurement.

From the obtained permeability coefficients for DPPC and Pad-PC-Pad it is not apparent that the amidophospholipid bilayers are interdigitated. However, it can be observed that CFloaded LUVs of Pad-PC-Pad quench to a higher degree than DPPC vesicles, indicating that the total proton influx is higher for Pad-PC-Pad liposomes.
SAXS Measurements of Pad-PC-Pad Dispersions. Small angle X-ray scattering measurements of Pad-PC-Pad aqueous dispersions were performed to investigate the interlamellar spacing of Pad-PC-Pad bilayers.

Figure 8 shows the scattering patterns of a hydrated Pad-PCPad sample at 21 and $40{ }^{\circ} \mathrm{C}$, which corresponds to

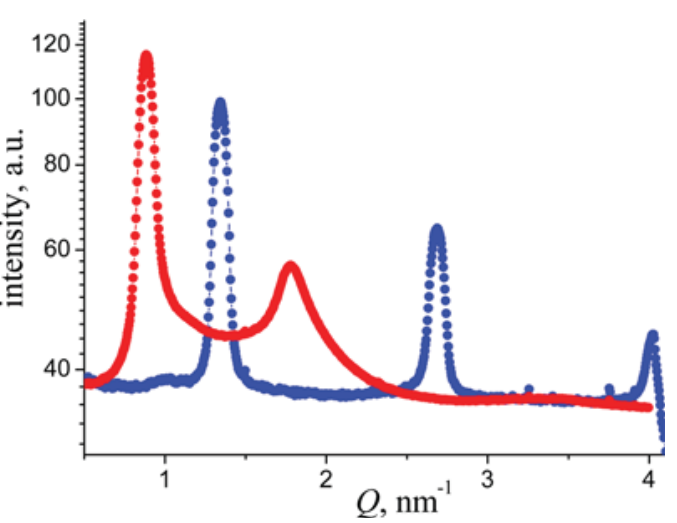

Figure 8. SAXS pattern of an aqueous dispersion of Pad-PC-Pad at 21 ${ }^{\circ} \mathrm{C}$ (blue curve) and at $40{ }^{\circ} \mathrm{C}$ (red curve).

measurements below and above the main phase transition temperature $\left(\sim 37{ }^{\circ} \mathrm{C}\right)$. The settings and preparation of the sample can be found in the Supporting Information.

In the gel phase at $21^{\circ} \mathrm{C}$, three Bragg peaks in a ratio of $Q_{1}: Q_{2}: Q_{3}=1: 2: 3$ typical for lamellar ordering can be clearly observed. The corresponding $d$-value amounts to $4.683 \mathrm{~nm}$. The quite sharp diffraction peaks indicate a good correlation between the bilayers. The correlation length $\xi$ amounts to $\sim 66$ $\mathrm{nm}$ corresponding to 14 well-correlated bilayers. The $d$-value includes the lipid bilayer thickness and the thickness of the water layer between the bilayers. The value is extremely small compared to the standard phospholipid 1,2-DPPC with $6.2 \mathrm{~nm}$. In the DPPC case, the chains are tilted by around $30^{\circ}$ to the bilayer normal. A larger tilt of the chains and a much smaller water layer between the bilayers cannot explain the observed small $d$-value. Therefore, interdigitation of the chains has to be taken into account. This situation is very similar to the one observed in 1,3-DPPC dispersions. ${ }^{34,46} 1,3-\mathrm{DPPC}$ forms an interdigitated lamellar phase with hexagonal chain packing and a very similar $d$-value of $4.7 \mathrm{~nm}$.

Above the chain melting transition, the SAXS pattern at 40 ${ }^{\circ} \mathrm{C}$ shows two broad diffraction peaks in a 1:2 ratio, indicating the appearance of a lamellar $\mathrm{L}_{\alpha}$ phase. The $d$-value is now 7.05 $\mathrm{nm}$, significantly larger than at $21^{\circ}$, showing that the chains in the molten state are not longer interdigitated and that the water layer between the bilayers must be thicker. This $d$-value is also larger than the one for the $\mathrm{L}_{\alpha}$ phase of 1,3-DPPC $(6.5 \mathrm{~nm})$. The correlation length $\xi$ in the $\mathrm{L}_{\alpha}$ phase is with $\sim 55 \mathrm{~nm}(8$ well-correlated bilayers) much smaller. Additionally, the increased intensity between the Bragg peaks could arise from uncorrelated bilayers or unilamellar vesicles formed above the melting transition.

\section{CONCLUSIONS}

It was shown that 1,3-diamidophospholipids could be formulated into vesicles that are faceted below their transition temperature. Careful selection of the method applied to form vesicles is necessary in order to avoid lipid degradation during 
vesicle formation. Method of formation and cooling rate are thus of significance for obtaining faceted vesicles.

Measurement of the proton permeability by fluorescence quenching indicates a rate constant similar to DPPC and PadPC-Pad, but with a higher permeability for the amidophospholipid, which can be explained by the fact that lipid chains of 1,3-diamidophospholipids are spread further apart than the chains of natural 1,2-phospholipids. Additionally, 1,3-diamidophospolipids possibly show strong hydrogen-bonding interactions between their headgroups. It has been suggested that passive proton transport across lipid bilayers is mediated by hydrogen-bonded chains of water, which could explain the increased total permeability. ${ }^{47,48}$

SAXS measurements confirm that Pad-PC-Pad forms interdigitated bilayers below the main transition temperature, a feature that disappears above the main transition temperature leading to a lamellar $\mathrm{L}_{\alpha}$ phase and a thicker water layer between the bilayers. This is in good agreement with the observation that the critical temperature $T_{\mathrm{c}}$ of a Pad-PC-Pad monolayer is much lower than the $T_{\mathrm{m}}$ of the corresponding bilayer system. ${ }^{23}$ The interdigitation in bilayers is the stabilizing factor leading to a $T_{\mathrm{m}}$ value larger than $T_{\mathrm{c}}$. The 1,3-motive is obviously the driving force for membrane interdigitation leading to a high lateral membrane stability, which suggests that vesicles formulated from 1,3-diamidophospholipids are prone to show facets. We argue that the faces of the facets would consist of rigid membrane segments and the vertices would correspond to a fluid phase. Such an arrangement could lead to instabilities at the vesicle vertices and would also explain why 1,3diamidophospholipid vesicles release an entrapped cargo upon the application of an external shear field.

Artificial 1,3-diamidophospholipids have strong potential for the formulation of liposomes that are sensitive to shear stress. This is necessary for the release of an entrapped cargo and could be easily controlled by the composition of lipids, which in turn influences the tendency to form more or less faceted vesicles.

\section{ASSOCIATED CONTENT}

\section{Supporting Information}

Additional experimental details.

\section{AUTHOR INFORMATION}

\section{Corresponding Authors}

*E-mail: brezesinski@mpikg.mpg.de.

*E-mail: marques@unistra.fr.

*E-mail: andreas.zumbuehl@unifr.ch.

\section{Notes}

The authors declare no competing financial interest.

\section{ACKNOWLEDGMENTS}

This work was supported by the Swiss National Science Foundation (Grants PP00P2_138926/1 and 200020_132035 to A.Z.) and European Cooperation in Science and Technology Action CM1101. The authors thank the Bioimaging, MS, and NMR facilities at the University of Geneva for analytical services. Cryo-EM was carried out in EMEZ (EM center of ETH Zurich).

\section{REFERENCES}

(1) Gregoriadis, G.; Leathwood, P. D.; Ryman, B. E. Enzyme entrapment in liposomes. FEBS Lett. 1971, 14 (2), 95-99.

(2) Bangham, A. D.; Standish, M. M.; Watkins, J. C. Diffusion of univalent ions across the lamellae of swollen phospholipids. J. Mol. Biol. 1965, 13 (1), 238-52.

(3) Immordino, M. L.; Dosio, F.; Cattel, L. Stealth liposomes: review of the basic science, rationale, and clinical applications, existing and potential. Int. J. Nanomed. 2006, 1 (3), 297-315.

(4) Mellal, D.; Zumbuehl, A. Exit-strategies-smart ways to release phospholipid vesicle cargo. J. Mater. Chem. 2014, 2 (3), 247-252.

(5) Holme, M. N.; Fedotenko, I. A.; Abegg, D.; Althaus, J.; Babel, L.; Favarger, F.; Reiter, R.; Tanasescu, R.; Zaffalon, P. L.; Ziegler, A.; Muller, B.; Saxer, T.; Zumbuehl, A. Shear-stress sensitive lenticular vesicles for targeted drug delivery. Nat. Nanotechnol. 2012, 7 (8), 536543.

(6) Fedotenko, I. A.; Zaffalon, P. L.; Favarger, F.; Zumbuehl, A. The synthesis of 1,3-diamidophospholipids. Tetrahedron Lett. 2010, 51 (41), 5382-5384.

(7) Dubois, M.; Zemb, T. Swelling limits for bilayer microstructures: The implosion of lamellar structure versus disordered lamellae. Curr. Opin. Colloid Interface Sci. 2000, 5 (1-2), 27-37.

(8) Blaurock, A. E.; Gamble, R. C. Small phosphatidylcholine vesicles appear to be faceted below the thermal phase-transition. J. Membr. Biol. 1979, 50 (2), 187-204.

(9) Lichtenberg, D.; Freire, E.; Schmidt, C. F.; Barenholz, Y.; Felgner, P. L.; Thompson, T. E. Effect of surface curvature on stability, thermodynamic behavior, and osmotic activity of dipalmitoylphosphatidylcholine single lamellar vesicles. Biochemistry 1981, 20 (12), 34623467.

(10) Lichtenberg, D.; Schmidt, C. F. Molecular packing and stability in the gel phase of curved phosphatidylcholine vesicles. Lipids 1981, 16 (7), 555-557.

(11) Sackmann, E. Membrane bending energy concept of vesicleshape and cell-shape and shape-transitions. FEBS Lett. 1994, 346 (1), $3-16$.

(12) Bagatolli, L. A.; Gratton, E. Two-photon fluorescence microscopy observation of shape changes at the phase transition in phospholipid giant unilamellar vesicles. Biophys. J. 1999, 77 (4), 20902101.

(13) Dubois, M.; Deme, B.; Gulik-Krzywicki, T.; Dedieu, J. C.; Vautrin, C.; Desert, S.; Perez, E.; Zemb, T. Self-assembly of regular hollow icosahedra in salt-free catanionic solutions. Nature 2001, 411 (6838), 672-675.

(14) Uchegbu, I. F.; Schatzlein, A.; Vanlerberghe, G.; Morgatini, N.; Florence, A. T. Polyhedral non-ionic surfactant vesicles. J. Pharm. Pharmacol. 1997, 49 (6), 606-10.

(15) Marques, E. F.; Regev, O.; Khan, A.; Miguel, M. d. G.; Lindman, B. Interactions between catanionic vesicles and oppositely charged polyelectrolytes phase behavior and phase structure. Macromolecules 1999, 32 (20), 6626-6637.

(16) Vernizzi, G.; de la Cruz, M. O. Faceting ionic shells into icosahedra via electrostatics. Proc. Natl. Acad. Sci. U.S.A. 2007, 104 (47), 18382-18386.

(17) Vernizzi, G.; Sknepnek, R.; de la Cruz, M. O. Platonic and Archimedean geometries in multicomponent elastic membranes. Proc. Natl. Acad. Sci. U.S.A. 2011, 108 (11), 4292-4296.

(18) Sknepnek, R.; Vernizzi, G.; de la Cruz, M. O. Buckling of multicomponent elastic shells with line tension. Soft Matter 2012, 8 (3), 636-644.

(19) Guo, H. X.; de la Cruz, M. O., Compartmentalization and delivery via asymmetric copolymer monolayers with swollen or inverse swollen micelles. J. Chem. Phys. 2010, 132 (9).

(20) Bealle, G.; Jestin, J.; Carriere, D. Osmotically induced deformation of capsid-like icosahedral vesicles. Soft Matter 2011, 7 (3), 1084-1089.

(21) Quemeneur, F.; Quilliet, C.; Faivre, M.; Viallat, A.; Pepin-Donat, B. Gel phase vesicles buckle into specific shapes. Phys. Rev. Lett. 2012, 108 (10). 
(22) Gonzalez-Perez, A.; Schmutz, M.; Waton, G.; Romero, M. J.; Krafft, M. P. Isolated fluid polyhedral vesicles. J. Am. Chem. Soc. 2007, 129 (4), 756-7.

(23) Fedotenko, L. A.; Stefaniu, C.; Brezesinski, G.; Zumbuehl, A. Monolayer properties of 1,3-diamidophospholipids. Langmuir 2013, 29 (30), 9428-35.

(24) Lobbecke, L.; Cevc, G. Effects of short-chain alcohols on the phase behavior and interdigitation of phosphatidylcholine bilayer membranes. Biochim. Biophys. Acta 1995, 1237 (1), 59-69.

(25) Vierl, U.; Lobbecke, L.; Nagel, N.; Cevc, G. Solute effects on the colloidal and phase behavior of lipid bilayer membranes: ethanoldipalmitoylphosphatidylcholine mixtures. Biophys. J. 1994, 67 (3), $1067-79$.

(26) Wang, Y.; Dea, P. Interaction of 1-propanol and 2-propanol with dipalmitoylphosphatidylcholine bilayer: A calorimetric study. J. Chem. Eng. Data 2009, 54 (5), 1447-1451.

(27) Reeves, M. D.; Schawel, A. K.; Wang, W.; Dea, P. Effects of butanol isomers on dipalmitoylphosphatidylcholine bilayer membranes. Biophys. Chem. 2007, 128 (1), 13-18.

(28) McIntosh, T.; McDaniel, R.; Simon, S. Induction of an interdigitated gel phase in fully hydrated phosphatidylcholine bilayers. Biochim. Biophys. Acta, Biomembr. 1983, 731 (1), 109-114.

(29) Hata, T.; Matsuki, H.; Kaneshina, S. Effect of local anesthetics on the phase transition temperatures of ether-and ester-linked phospholipid bilayer membranes. Colloids Surf., B 2000, 18 (1), 4150 .

(30) Schmutz, M.; Michels, B.; Marie, P.; Krafft, M. P. Fluorinated vesicles made from combinations of phospholipids and semifluorinated alkanes. Direct experimental evidence of the location of the semifluorinated alkane within the bilayer. Langmuir 2003, 19 (12), 4889-4894.

(31) Huang, C. Mixed-chain phospholipids and interdigitated bilayer systems. J. Mol. Med. 1990, 68 (3), 149-65.

(32) Huang, C.; Mason, J. T. Structure and properties of mixed-chain phospholipid assemblies. Biochim. Biophys. Acta 1986, 864 (3-4), 42370.

(33) Smith, E. A.; Dea, P. K. Differential scanning calorimetry studies of phospholipid membranes: The interdigitated gel phase. In Applications of Calorimetry in a Wide Context-Differential Scanning Calorimetry, Isothermal Titration Calorimetry and Microcalorimetry; Intech: Rijeka, Croatia, 2013; pp 407-44.

(34) Serrallach, E. N.; Dijkman, R.; de Haas, G. H.; Shipley, G. G. Structure and thermotropic properties of 1,3-dipalmitoyl-glycero-2phosphocholine. J. Mol. Biol. 1983, 170 (1), 155-74.

(35) Ishikawa, T.; Sakakibara, H.; Oiwa, K. The architecture of outer dynein arms in situ. J. Mol. Biol. 2007, 368 (5), 1249-1258.

(36) Rothemund, P. W. K. Folding DNA to create nanoscale shapes and patterns. Nature 2006, 440, 297-302.

(37) Sawayama, J.; Sakaino, H.; Kabashima, S.-i.; Yoshikawa, I.; Araki, K. Hydrogen-bond-directed giant unilamellar vesicles of guanosine derivative: Preparation, properties, and fusion. Langmuir 2011, 27, 8653-8658

(38) Menger, F. M.; Angelova, M. I. Giant vesicles: Imitating the cytological processes of cell membranes. Acc. Chem. Res. 1998, 31, 789-797.

(39) Morales-Penningston, N. F.; Wu, J.; Farkas, E. R.; Goh, S. L.; Konyakhina, T. M.; Zheng, J. Y.; Webb, W. W.; Feigenson, G. W. GUV preparation and imaging: Minimizing artifacts. Biochim. Biophys. Acta 2010, 1798, 1324-1332.

(40) Israelachvili, J. N. Intermolecular and Surface Forces, revised 3rd ed.; Academic Press: New York, 2011.

(41) Horger, K. S.; Estes, D. J.; Capone, R.; Mayer, M. Films of agarose enable rapid formation of giant liposomes in solutions of physiologic ionic strength. J. Am. Chem. Soc. 2009, 131 (5), 1810-9.

(42) Weinberger, A.; Tsai, F. C.; Koenderink, G. H.; Schmidt, T. F.; Itri, R.; Meier, W.; Schmatko, T.; Schroder, A.; Marques, C. Gelassisted formation of giant unilamellar vesicles. Biophys. J. 2013, 105 (1), 154-64.
(43) Zeng, J.; Smith, K. E.; Chong, P. L. Effects of alcohol-induced lipid interdigitation on proton permeability in L-alpha-dipalmitoylphosphatidylcholine vesicles. Biophys. J. 1993, 65 (4), 1404-14.

(44) Komatsu, H.; Okada, S. Increased permeability of phaseseparated liposomal membranes with mixtures of ethanol-induced interdigitated and non-interdigitated structures. Biochim. Biophys. Acta 1995, 1237 (2), 169-75.

(45) Komatsu, H.; Chong, P. L. Low permeability of liposomal membranes composed of bipolar tetraether lipids from thermoacidophilic archaebacterium Sulfolobus acidocaldarius. Biochemistry 1998, 37 (1), 107-15.

(46) Stümpel, J.; Eibl, H.; Nicksch, A. X-ray analysis and calorimetry on phosphatidylcholine model membranes. The influence of length and position of acyl chains upon structure and phase behaviour. Biochim. Biophys. Acta, Biomembr. 1983, 727 (2), 246-254.

(47) Nagle, J. F.; Tristram-Nagle, S. Hydrogen bonded chain mechanisms for proton conduction and proton pumping. J. Membr. Biol. 1983, 74 (1), 1-14.

(48) Deamer, D. W.; Nichols, J. W. Proton flux mechanisms in model and biological membranes. J. Membr. Biol. 1989, 107 (2), 91-103. 